\title{
Transmissão de risco entre os índices setoriais do Ibovespa: uma aplicação do teste de causalidade em variância
}

\author{
Helberte João França Almeida ${ }^{1}$ \\ José Emanuel Camargo Neto ${ }^{2}$ \\ Adilson Giovanini3 \\ Kleverton Clovis Oliveira Saath 4
}

\begin{abstract}
Resumo: A atual globalização financeira permite uma forte ligação entre os mercados e uma alta velocidade de deslocamento do capital. No caso de países emergentes, esse deslocamento é intensificado de maneira regional, ou seja, entre os setores do próprio país. Assim, o presente estudo busca avaliar a transmissão de risco entre os índices setoriais do Ibovespa utilizando a abordagem de causalidade em variância. Os resultados obtidos mostram que a causalidade entre os ativos é, em geral, bidirecional, o que vai ao encontro da teoria. Além disso, em momentos de crise há maior volatilidade entre os ativos financeiros, proporcionando o aumento de transmissão de risco entre os mercados.
\end{abstract}

Palavras-chave: Causalidade na variância; risco; volatilidade; índices setoriais.

Transmission of risk among Ibovespa sector indices: an application of the causality test in variance.

\begin{abstract}
Current financial globalization allows for a strong connection between markets and high speed capital flow. In the case of emerging countries, this flow is intensified regionally, that is, between the country's own sectors. Therefore, this study aims to investigate whether there is risk transmission between Ibovespa's sector indices using the causality in variance approach. The obtained results show there is causality between assets is, in general, bidirectional, which corroborates established theory. Additionally, in moments of crisis there is greater volatility between financial assets, increasing risk transmission between markets.
\end{abstract}

\footnotetext{
${ }^{1}$ Prof. Dr. da Universidade Federal de Santa Catarina, UFSC. E-mail: helberte.almeida@ufsc.br.

2 Graduado Economia, Universidade Federal de Santa Catarina. UFSC, E-mail: zecamargoneto@gmail.com 3 Prof. Dr. da Universidade do Estado de Santa Catarina, UDESC. E-mail: adilsoneconomia@gmail.com 4 Mestre Economia, Universidade Federal de Santa Catarina, UFSC. E-mail: klevertonsaath1995@hotmail.com
} 
Keywords: Causality in variance; risk; volatility; sector indices;

Classificação JEL: F40; G10; G20; G30.

\section{Introdução}

O aumento de possibilidades de investimentos e o elevado nível de globalização em que os mercados financeiros se encontram acabam por ocasionar um aumento da correlação entre os retornos apresentados pelos ativos que os compõem. Como salienta Vo (2009), essa proximidade entre os mercados facilita a mobilidade de capitais e, consequentemente, o mercado irá se mover conjuntamente, assim, tal movimento ocasiona maior contágio financeiro e o aumento da volatilidade de um mercado para outro. Segundo Cintra (1998), o fluxo de capitais - tanto nacional como internacional - por ter mais opções, busca os melhores retornos e isso gera um significativo aumento de volatilidade nas operações.

A elevação da volatilidade pode ser traduzida por contágio financeiro, e se manifesta como um aumento significativo da volatilidade apresentado pelos retornos de dois ou mais mercados após um choque em um mercado específico. $\mathrm{O}$ aumento da volatilidade em um dado mercado acaba influenciando no comportamento da volatilidade apresentada pelos demais mercados, fazendo com que se observe um aumento geral da volatilidade em ambos os mercados. Assim, a ocorrência de períodos de volatilidade elevada em um desses mercados pode levar os investidores a realocar a sua carteira (por aversão ao risco ou mesmo por novas oportunidades de retorno), fazendo com que o aumento da volatilidade de um ativo seja transmitido para outros mercados e vice-versa (Forbes e Rigobon, 2002).

Uma vasta quantidade de estudos tem surgido na literatura econômica para avaliar os efeitos da transmissão da volatilidade no mercado financeiro. Uma linha de pesquisa nesta análise é formada por autores que buscam avaliar os efeitos da volatilidade no mercado acionário e reúne autores como Cheung e Ng (1996), Hu et al. (1997) e Pantelidis e Pittis (2004). A conclusão geral alcançada por estes é que existe transmissão de volatilidade entre os mercados, sendo que o sentido em que ocorre a transmissão da volatilidade pode ser unidirecional ou bidirecional.

Além desses trabalhos, existem outros estudos que buscam avaliar a volatilidade do mercado financeiro em momentos de crise. Assim, os trabalhos de Forbes e Rigobon (2002) e Righi e Ceretta (2013) encontram como resultado que existe um forte aumento de correlação entre os ativos e, consequentemente, um aumento da volatilidade nos períodos de crise. 
Embora a literatura econômica reconheça a existência de transmissão de volatilidade entre os ativos, e a influência desse efeito sob as variáveis macroeconômicas, a maioria dos estudos está relacionado a identificar a estrutura de dependência entre os países distintos ou testar a ocorrência de mudanças (decorrentes de crises financeiras) nessa estrutura. Assim, existe uma lacuna na literatura de finanças que diz respeito à existência de canais de propagação de volatilidade entre agrupamentos menores de ativos financeiros.

A motivação para o presente estudo são os resultados encontrados por Baca et al. (2000) e Phylaktis et al. (2011). Em seu estudo, os primeiros alcançam o resultado que os movimentos setoriais têm maior poder de explicação da volatilidade quando comparado ao movimento dos próprios países em sete importantes mercados de ações no mundo. Por sua vez, Phylaktis et al. (2011) encontram o resultado que os setores das economias asiáticas são mais correlacionados com o desempenho da economia norte-americana e os mercados da América Latina apresentam-se de maneira mais dependente da economia local, ou seja, são mais correlacionados com os índices regionais de mercado.

Dessa forma, a contribuição do presente estudo para a literatura de finanças é verificar se existe transmissão de volatilidade entre os índices setoriais do Ibovespa. Para tanto, utiliza-se a abordagem proposta por Cheung e Ng (1996), ou seja, utiliza-se o teste de causalidade em variância. $O$ trabalho encontra-se estruturado em quatro seções, além desta breve introdução. A seção dois apresenta o referencial teórico e as principais contribuições relacionadas ao tema do trabalho. Por sua vez, a seção três traz o procedimento metodológico adotado. $\mathrm{Na}$ seção quatro encontram-se as variáveis utilizadas, bem como os resultados obtidos e sua discussão. Por fim, a seção cinco expõe as considerações finais.

\section{Revisão de literatura}

O investidor racional, segundo o princípio da dominância (Sharpe et al., 1995, p.194), busca alocar seu capital em ativos que possam lhe garantir maiores retornos mediante um dado risco. Assim, a chamada especulação racional tem por premissa que os preços são formados com base nas expectativas de retorno dos mercados futuros (Copeland et al., 1998).

Nesse ambiente de maximização de retorno, o comportamento estocástico de correlações e covariâncias entre os retornos dos ativos é de profunda importância para a precificação e seleção de ativos para uma carteira, a fim de se administrar o risco e precificar os ativos de maneira coerente (Caporin e Mcaller, 2010). Tradicionalmente, o cálculo de estimativas referente à volatilidade de retornos financeiros é baseado na variação diária no movimento de preços dos 
ativos (Goodhart e O'hara, 1997). Dessa forma, diversos modelos distintos foram e têm sido desenvolvidos com a finalidade de tratar esses movimentos.

De maneira geral, o modelo mais simples que se tem é o desvio padrão histórico que, por sua vez, adiciona pesos uniformes a todas as observações. Por se tratar de um modelo simples, sua aplicabilidade se torna alta, porém, torna-se precário por ser simétrico e uniforme (constante) em todas as observações (Goodhart e O'hara, 1997). Outra vertente que surge com o intuito de sanar esses problemas e modelar a volatilidade (movimento nos preços) em séries históricas financeiras é introduzida por Engle (1982): os modelos ARCH (Autoregressive Conditional Heteroscedasticity) e sua generalização, o modelo GARCH (Generalized Autoregressive Conditional Heteroscedasticity), proposta por Bollerslev (1986). Esses modelos tem por característica uma forma simples e eficaz de modelar a volatilidade de preços.

Devido à globalização financeira, a volatilidade entre os ativos tem aumentado e os investidores, na tentativa de garantir maior retorno às suas carteiras de investimento, acabam gerando maior volatilidade que poderá ocasionar uma crise financeira. Nesse contexto, Rigobon (2002), em uma de suas pesquisas (International Financial Contagion: Theory and Evidence in Evolution), descreve diversos casos de crises que ocorreram durante a amplitude das crises, ou seja, observar quem as causou e quem sofreu com ela.

\footnotetext{
Many people believe that every time one developing country sneezes, the rest of the world's emerging markets are likely to suffer from acute pneumonia. No matter how good their fiscal accounts are or how balanced the external accounts look, all countries are affected, at least to some degree, when a financial crisis hits somewhere across the globe (Rigobon, 2002).
}

Nessa explanação, o autor apresenta o caso presenciado pelo Brasil chamado internacionalmente de Brasilian Sneeze (o "espirro brasileiro"), ocorrido em 1999, com a desvalorização do real, em que ele expõe que tal crise afetou apenas o mercado interno, sem se propagar demasiadamente (por mais que a Argentina tenha sofrido muito).

Essa transmissão de volatilidade entre mercados ou, em outras palavras, esse contágio financeiro, ocorre em momentos de crises internacionais. Contudo, segundo Almeida (200o), é necessário que se determine corretamente se esses momentos são de contágio, ou apenas momentos de interdependência, em razão das relações existentes entre os mercados serem bem interligados. Co-movimentos entre mercados podem acontecer mesmo durante os tempos de tranquilidade, sendo esse um movimento de interdependência e não de transmissão. O contágio implica necessariamente em uma mudança nas ligações existentes entre os 
mercados, e essa mudança deve gerar uma elevação da interligação entre eles e, junto, uma elevação da volatilidade (Almeida, 2000).

Diante disto, Righi e Ceretta (2013), desenvolvem um estudo em que demonstram, através de um modelo GARCH multivariado com parametrização BEKK, o comportamento da volatilidade do índice brasileiro (Ibovespa) com relação a outros mercados. Em seu estudo, os autores analisam o mercado americano, argentino, mexicano e chinês. Com cerca de 10 anos de dados diários, sua análise é dividida em 3 períodos que compreendem a crise subprime de 2007/2008 (os períodos são: antes, durante e depois da crise). Os autores concluem que em períodos de crise há uma elevação da volatilidade dos mercados e, no caso brasileiro, essa elevação de risco gera uma mudança na direção da causalidade.

Estudos sobre a volatilidade dos ativos e a sua dinâmica temporal têm sido abordados desde antes de Lo e Mackinlay (2015), porém são os mesmos que observam a existência de uma correlação cruzada entre as ações. É demonstrado que essas correlações seriam assimétricas, ou seja, uma volatilidade seria a causadora da outra. No entanto, outras vertentes teóricas têm mostrado que ao utilizar dados voláteis e instáveis, típicos de economias emergentes, seria melhor utilizar a causalidade de segunda ordem, ou seja, causalidade em variância (Forbes e Rigobon, 2002).

Segundo Cheung e Ng (1996), a transmissão de volatilidade pode ser captada por meio da análise na causalidade em variância, visto que essa variação é tida como uma maneira de refletir a chegada de novas informações e também são capazes de medir a extensão em que os mercados avaliam e assimilam essas informações. Fama (1965) afirma que, em uma economia sem arbitragem, a variância dos preços está diretamente relacionada com a taxa de fluxo de informações para o mercado. Fama (1970), atribuem essa alteração na variância como sendo o tempo requerido pelos participantes do mercado no processamento de novas informações ou na coordenação de políticas. Assim, a relação entre fluxo de informação e volatilidade oferece uma perspectiva interessante para interpretar a causalidade em variância entre séries temporais econômicas. Por sua vez, o padrão de causalidade na variância fornece uma visão sobre as características e dinâmica dos preços econômicos e financeiros, e tal informação pode ser usada para construir modelos econométricos melhores, descrevendo a dinâmica temporal das séries.

Os estudos de Caporale et al. (2002), Van Dijk et al. (2005), Pantelidis e Pittis (2004) e Bhar e Hamori (2005), utilizando a abordagem de Cheung e Ng (1996), realizam o teste de causalidade em variância para avaliar as séries estudadas, em grande parte a relação entre os mercados de ativos (ações), em dados de alta e baixa frequência diários de diferentes países. A conclusão geral alcançada por esses 
autores é que existe transmissão de volatilidade entre os mercados e que o sentido encontrado é em grande parte bidirecional.

$\mathrm{Na}$ linha de estudos sobre propagação de volatilidade entre ativos financeiros menores, encontram-se Righi e Ceretta (2013). Os autores mostram que há transmissão de volatilidade entre pares de índices brasileiros. Assim, é possível corroborar seu estudo com as hipóteses de que a transmissão em países emergentes realmente existe e de maneira intersetorial (no caso de seu estudo, a transmissão ocorreu de maneira unidirecional - partindo do índice Mid-Large Cap para o índice Small-Cap - que são compostos pelas ações de grande e pequena capitalização, respectivamente).

\section{Metodologia}

Com a finalidade de observar a transmissão da volatilidade entre os índices que compõem o Ibovespa, o presente trabalho utilizar-se-á da modelagem proposta por Cheung e Ng (1996), causalidade na variância, e por meio das análises de Phylaktis et al. (2011), no que tange ao fato de tratar-se do mercado brasileiro e, consequentemente, latino-americano, propõe-se avaliar se essa transmissão ocorre de maneira setorial.

\subsection{Procedimentos Metodológicos}

O teste de causalidade em variância é sensível à presença de causalidade em média. Por esse motivo é escolhido o modelo Arma (Autoregressivo com Médias Móveis) para filtrar a relação na média existente entre as variáveis (TSAY, 2012). Formalmente:

$$
r_{t}=\emptyset+\sum_{i=1}^{p} \emptyset_{i} r_{t-1}+a_{t}-\sum_{i=1}^{q} \theta_{i} a_{t-1},
$$

sendo $r_{t}$ o retorno do ativo no período $t ; a_{t}$ é o ativo com média zero e variância $1 ; p$ identifica as defasagens do retorno do ativo.

O modelo VAR (Vetor Autoregressivo) é utilizado para identificar a relação existente entre as variáveis financeiras e econômicas consideradas. Dessa forma, é empregada a metodologia desenvolvida por Toda (1995), que apresenta uma alternativa ao modelo VAR tradicional, capaz de contornar o fato de ocorrer perdas de informações relevantes, e tornar as variáveis estacionárias. Precisamente:

$$
I_{t}=\alpha_{0}+\alpha_{k_{1}} \sum_{k=1}^{p} I_{t-k}+\alpha_{k_{2}} \sum_{k=1}^{p} i_{t-1}^{e}+\alpha_{k_{\mathrm{g}}} \sum_{k=1}^{p} D_{e_{t}-k}+\alpha_{k_{4}} \sum_{k=1}^{p} S_{t_{-k}}^{e}+\epsilon_{t}
$$


em que $i_{t}$ é um vetor composto pelos índices setoriais do Ibovespa descritos acima nas variáveis, representado pelo período $t-k$; $_{i e e_{t-k}}$ denota o Índice de Energia Elétrica; $i n d x_{t-k}$ é o Índice Industrial; $i c o n_{t-k}$ é Índice de Consumo; $i m o b_{t-k}$ denota o Índice Imobiliário; imat $_{t-k}$ é o Índice de Materiais Básicos; $u t i l_{t-k}$ é o Índice de Utilidade Pública; ibov $_{t-k}$ é o Ibovespa; if $_{n x_{t-k}}$ denota o Índice Financeiro. Além dos índices setoriais, adiciona-se ao modelo VAR estimado os seguintes indicadores macroeconômicos: $i_{t-1}^{e}$ que pondera os desvios da taxa de juros em relação às expectativas do mercado; $S^{e}$ que pondera os desvios da taxa de câmbio em relação às expectativas do mercado; $D e_{t-k}$ que é uma variável binária para o câmbio que identifica a ocorrência de três períodos ou mais de desvalorização cambial.

O modelo proposto por Baba, Engle, Kraft e Kroner (BEKK), desenvolvido por Engle (1982) e o modelo Constant Conditional Correlation (CCC), proposto por BOLLERSLEV (1986), são utilizados para identificar o comportamento da volatilidade existente entre os índices setoriais selecionados. Esses modelos consideram que a volatilidade varia no tempo, permitindo identificar a volatilidade dos ativos financeiros. A forma condicional do modelo BEKK mais utilizado pela literatura para explicar a volatilidade dos ativos financeiros é o modelo BEKK $(\mathrm{p}, \mathrm{q}, \mathrm{k})$. Esse modelo é expresso por:

$$
\mathrm{H}_{\mathrm{t}}=\mathrm{C}^{\prime} \mathrm{C}+\sum_{\mathrm{q}=1}^{\mathrm{Q}} \mathrm{A}_{\mathrm{t}-\mathrm{q}}^{\prime} \in_{\mathrm{i}, \mathrm{t}-\mathrm{q}} \in_{\mathrm{j}, \mathrm{t}-\mathrm{q}}^{\prime} \mathrm{A}_{\mathrm{t}-\mathrm{q}}+\sum_{\mathrm{p}=1}^{\mathrm{p}} \mathrm{B}_{\mathrm{t}-\mathrm{p}}^{\prime} \mathrm{H}_{\mathrm{t}-\mathrm{p}} \mathrm{B}_{\mathrm{t}-\mathrm{p}}
$$

em que $C, A_{i}$ e $B_{i}$ são matrizes de parâmetros com dimensão $N x N$; $C$ é uma matriz triangular superior; e $H_{t}$ é a matriz de variância e covariância condicional. A forma matricial da variância condicional é definida por:

$$
\begin{gathered}
\mathrm{H}_{\mathrm{t}}=\left[\begin{array}{cc}
C_{11} & C_{12} \\
0 & C_{22}
\end{array}\right]+\left[\begin{array}{ll}
a_{11} & a_{12} \\
a_{21} & a_{22}
\end{array}\right]^{\prime} \cdot\left[\begin{array}{cc}
\in_{1, t-1}^{2} & \in_{1, t-1} \in^{\prime}, t-1 \\
\in_{1, t-1} \in_{2, t-1}^{\prime} & \in_{2, t-1}^{2}
\end{array}\right] \cdot\left[\begin{array}{ll}
a_{11} & a_{12} \\
a_{21} & a_{22}
\end{array}\right] \\
+\left[\begin{array}{ll}
g_{11} & g_{21} \\
g_{21} & g_{22}
\end{array}\right]^{\prime} \cdot\left[\begin{array}{cc}
h_{11, t-1} & h_{12, t-1} \\
h_{1, t-1} \in_{2, t-1}^{\prime} & h_{22, t-1}
\end{array}\right] \cdot\left[\begin{array}{ll}
g_{11} & g_{12} \\
g_{21} & g_{22}
\end{array}\right]
\end{gathered}
$$

O modelo BEKK capta a dependência dinâmica da volatilidade, mas apresenta diversas limitações, a saber: 1) conforme o número de ativos cresce, o número de parâmetros cresce rapidamente, dificultando a estimação do modelo; e 2) $a$ e $g$ não possuem interpretação direta. 
Uma alternativa ao modelo BEKK é o modelo de correlação condicional constante (CCC). Esse modelo restringe a matriz de correlações tornando-a constante. Sendo assim, somente os desvios condicionais são variáveis no tempo. $\mathrm{O}$ modelo CCC pode ser definido conforme a formulação. Ele é obtido a partir da decomposição de Cholesck $\left(\sum t=L_{t} G L_{t}\right)$ da matriz de covariâncias e é definido por:

$$
\sum t=\alpha_{0}+\alpha_{1} \epsilon_{t-1}^{2}+\beta_{1} \sum t-1,
$$

no qual $\alpha_{1}$ e $\beta_{1}$ são matrizes não-negativas definidas e $\epsilon_{t}^{2}$ pode ser reescrito como um modelo ARMA(1,1) bivariado $\operatorname{com}_{n_{t}}=\mathrm{E}_{t}^{2}-\sum t$ :

$$
\epsilon_{\mathrm{t}}^{2}=\alpha_{0}+\left(\alpha_{1}+\beta_{1}\right) \epsilon_{\mathrm{t}-1}^{2}+\mathrm{n}_{\mathrm{t}}-\beta_{1} \mathrm{n}_{\mathrm{t}-1}
$$

A limitação apresentada pelo modelo CCC é que ele considera que a matriz de correlação entre os ativos permanece constante ao longo do tempo. Para contornar essa limitação, Tse e Tsui (200o) adotam o modelo de correlação condicional dinâmica (DCC). Formalmente:

$$
\sum \mathrm{t}=\mathrm{D}_{\mathrm{t}} \mathrm{R}_{\mathrm{t}} \mathrm{D}_{\mathrm{t}}
$$

em que $D_{t}$ é uma matriz diagonal com GARCH univariados; e $R_{t}$ é uma matriz de correlação dos resíduos padronizados.

$$
\mathrm{R}_{\mathrm{r}}=\left(1-\theta_{1}-\theta_{2}\right) \mathrm{R}_{\mathrm{t}}+\theta_{1} \Psi_{\mathrm{t}-1}+\theta_{2} \mathrm{R}_{\mathrm{t}-1}
$$

Por sua vez $\theta_{1}$ e $\theta_{2}$ são parâmetros não-negativos sujeitos à restrição $\theta_{1}+\theta_{2}<1$ e $\psi$ é uma matriz positiva definida:

$$
\Psi_{\mathrm{ijt}-1}=\frac{\sum_{\mathrm{m}=1}^{\mathrm{M}} \mathrm{U}_{\mathrm{it}}-\mathrm{u}_{\mathrm{jt}-\mathrm{m}}}{\sqrt{\left(\sum_{\mathrm{m}=1}^{\mathrm{M}} \mathrm{u}_{\mathrm{it}-\mathrm{m}}^{2}\right)\left(\sum_{\mathrm{m}=1}^{\mathrm{M}} \mathrm{u}_{\mathrm{jt}-\mathrm{m}}^{2}\right.}}
$$

na qual $u_{i t}=\frac{E_{\text {it }}}{h_{\text {it }}}$.

Um modelo semelhante $\sum t=D_{t} R_{t} D_{t}$, no qual $R_{t}$ é definido como uma matriz diagonal com GARCH univariados, enquanto $R_{t}$ é uma matriz de correlação dos resíduos padronizados com dinâmica: 


$$
\sum \mathrm{t}=\left(1-\sum_{1=1}^{\mathrm{L}} \alpha_{l}\right)-\left(\sum_{s=1}^{\mathrm{s}} \beta_{s} \sum_{1=1}^{\mathrm{L}} \alpha_{1}\right) \mathrm{u}_{\mathrm{t}-1} \mathrm{u}_{\mathrm{t}-1}^{\prime}+\sum_{s=1}^{\mathrm{s}} \beta_{s \Sigma \mathrm{t}-\mathrm{s}}
$$

sendo $\alpha_{l}$ e $\beta_{s}$ parâmetros não negativos que satisfazem a restrição $\sum_{l=1}^{L} \alpha_{1}+\sum_{s=1}^{S} \beta_{1}<1$ e Qé uma matriz de variância não condicional.

Essa metodologia se caracteriza pela dificuldade de mensuração da ordem adequada do AR e do MA para estimar o modelo ARMA $(p, q)$ e através dele estimar qual o modelo de volatilidade BEKK, CCC ou DCC que explica melhor a dinâmica apresentada pela volatilidade. A identificação das ordens AR e MA exige muita cautela porque uma estimação de um modelo $\operatorname{ARMA}(p, q)$ com ordens incorretas pode comprometer o ajuste do modelo e, por conseguinte, comprometer a análise da volatilidade. Ademais, conforme enfatizado por Cheung e Ng (1996), a causalidade em média precisa ser filtrada quando se pretende testar se existe causalidade em variância entre duas variáveis.

Os critérios de informação de Akaike (AIC) e o critério bayesiano de Schwarz (BIC) são utilizados para identificar a ordem AR e MA dos modelos ARMA $(p, q)$ que apresentam melhor ajuste. Dessa maneira, os critérios AIC e BIC são utilizados com o intuito de se escolher o número de defasagens empregadas na estimação do modelo.

$$
\begin{array}{r}
A I C_{p_{i} q}=\ln \Gamma(\Theta)+\frac{2(p+q)}{N} \\
B I C_{p, q}=\ln \Gamma(\Theta)+(\mathrm{p}+\mathrm{q}) \frac{\ln N}{N}
\end{array}
$$

$\Gamma(\Theta)$ é o estimador de máxima verossimilhança. Portanto, o modelo que apresentar os menores valores para os critérios de informação será o escolhido. Cheung e $\mathrm{Ng}$ (1996) desenvolveram um teste para detectar a presença de causalidade em variância. Para isso, consideram que dado o conjunto de ativos $y_{t}=\left(y_{1 t}, y_{2 t}\right)^{\prime}, y_{2 t}$ causa $y_{1 t}$ em variância se

$$
E\left[\left(y_{1 t}-\mu_{1 t}\right)^{2}\left|\Omega_{t-1}\right| \neq E\left[\left(y_{1 t}-\mu_{1 t}\right)^{2}\left|\Omega_{1 t-1}\right|\right.\right.
$$

em que $\Omega_{i t}$ representa o conjunto de informações, definido por $\Omega_{i t}=\left\{y_{i t-j} ; \leq 0\right\}, i=1,2, \Omega_{t}=\Omega_{i t} \cup \Omega_{2 t} \mathrm{e}_{\mu_{i t}}$ é a média de $y_{i t}$ condicional em $\Omega_{i t}$. Denotando-se $\epsilon_{i t}=y_{i t}-\mu_{i t}$ e considerando $\epsilon_{i t}=\xi_{i t} \sqrt{h_{i t}}$, em que $h_{i t}$ é uma função positiva variante no tempo, $\xi_{i t}$ uma inovação com $E\left[\xi_{i t}^{2} \mid \Omega_{i t-1}\right]=1$. Como $h_{i t}$, por 
construção, é a variância condicional de $\epsilon_{i t}$, ou seja, $h_{i t}=V\left[\epsilon_{i t} \mid \Omega_{i t-1}\right]$, então a hipótese nula de que $y_{2 t}$ não causa $y_{i t}$ em variância pode ser formulada por:

Ho: $V\left[\epsilon_{i t} \mid \Omega_{i t}\right]=V\left[\epsilon_{i t} \mid \Omega_{1 t-1}\right]$. O resíduo padronizado ao quadrado é definido como:

$$
v_{\mathrm{t}}=\frac{\left(\mathrm{y}_{1 \mathrm{t}}-\mu_{1 \mathrm{t}}\right)^{\mathrm{a}}}{\mathrm{h} r_{1 \mathrm{t}}}
$$

e a correlação cruzada para a defasagem $k$ como:

$$
r_{u v}(k)=\frac{c_{u v}}{\sqrt{c_{u u}(0) c_{v v}(0)}}
$$

em que $C_{w v}(k)$ é a covariância cruzada amostral, ou seja:

$C_{u v}(k)=\left\{\begin{array}{c}\frac{1}{T} \sum_{t=k+1}^{T}\left(u_{t}-u^{\prime}\right)\left(v_{t-k}-v^{\prime}\right), \text { se } k \leq 0 \\ (k)=\frac{1}{T} \sum_{t=1}^{T=k-1}\left(u_{t}-u^{\prime}\right)\left(v_{t-k}-v^{\prime}\right), \text { se } k<0\end{array}\right.$,

no qual $T$ é o tamanho da amostra, $u^{\prime}$ e $v^{r}$ são as médias amostrais de $u_{t} \mathrm{e}^{\mathrm{e}} v_{t}$ respectivamente, $\mathrm{e}_{C_{x x}}=\frac{1}{T} \sum_{t=1}^{T}\left(x_{t}-x^{I}\right)^{2}$ para $x=u, v$ é a variância.

Dessa forma, Ho pode ser testado por meio da estatística:

$$
\mathrm{S}=\mathrm{T} \sum_{\mathrm{k}=1}^{\mathrm{K}} \mathrm{R}_{\mathrm{uv}}^{2}(\mathrm{k})
$$

que possui distribuição assintótica qui-quadrado com $\mathrm{k}$ graus de liberdade. Contudo, Hong (2001) propôs duas modificações nesse teste: 1) ele considera que a média amostral $\mu_{i t}$ representa a média de $y_{i t}$ condicional para toda a informação $\left(\Omega_{t-1}\right)$ o que garante que a causalidade na média não influencia no teste de causalidade em variância. 2) sugere pesos para as correlações cruzadas para obter um teste mais poderoso:

$$
\mathrm{Q}=\frac{\mathrm{T} \sum_{\mathrm{k}=1}^{\mathrm{T}-1} \mathrm{w}^{2}(\mathrm{k} ; \mathrm{K}) \mathrm{r}_{\mathrm{u}}^{\mathrm{x}}(\mathrm{l})-\mathrm{C}(\mathrm{w})}{\sqrt{2 \mathrm{D}(\mathrm{w})}} \sim \mathrm{N}(0,1)
$$

em que $w(k ; K)$ representa uma função peso Bartlett Kernel 
$C_{w}=\left\{\begin{array}{c}1-\left|\frac{k}{k+1} ;\right| \text { se } \frac{K}{k+1} \leq 1 \\ 0 ; \text { caso contrario, }\end{array}\right.$

sendo

$$
\mathrm{C}_{\mathrm{w}}=\frac{\sum_{\mathrm{k}}^{\mathrm{I}-1}(1-\mathrm{k}]}{\mathrm{Tw}^{2}(\mathrm{k} ; \mathrm{K})}
$$

e $\quad \mathrm{D}(\mathrm{w})=\sum_{K=1}^{T-1}\left(1-\frac{\mathrm{k}}{\mathrm{T}}\right)\left(1-\frac{\mathrm{k}+1}{\mathrm{Tw}^{4}(\mathrm{k} ; \mathrm{K})}\right)^{\prime}$

os quais são aproximadamente a média e a variância de $\sum_{k=1}^{t-1} w^{2}(k ; K) r_{u v}^{2}(k)$.

\subsection{Desvios das expectativas: uma pequena digressão}

O presente estudo incorpora as expectativas dos agentes em relação à taxa de juros e o câmbio futuro como variáveis que afetam a transmissão de volatilidade entre os índices do Ibovespa. Contudo, por meio do levantamento, as expectativas dos juros futuros podem ser classificadas em otimistas, pessimistas ou neutras, sendo representadas por uma expectativa de juros futuros maiores, menores ou iguais aos da data vigente.

O quadro 1 apresenta como se comportam as expectativas do mercado (obtida do boletim Focus) e utilizado no modelo que, por sua vez, trabalha ponderando os desvios das taxas efetivas do período com as expectativas dos agentes e, dessa maneira, possuindo uma resposta binária, que é a de manter ou não a mesma expectativa passada no período seguinte.

Assim, o quadro 1 expressa um indivíduo no mercado e sua expectativa com relação à taxa de juros futura. No período 1, esse agente possui uma expectativa neutra com relação às taxas para o futuro, porém a taxa efetiva é menor que a anterior (caindo de $11 \%$ para $10 \%$ ). Dessa maneira, a expectativa de taxas futuras do indivíduo se altera, mudando de $11 \%$ para $10,5 \%$. Já no período seguinte, a taxa encontrada continua reduzindo, de $10 \%$ para $9 \%$, ao contrário do que esperavam os investidores. Contudo, a expectativa para o período seguinte muda novamente, dessa vez, para 9,5\% pois ainda acredita num aumento de taxa (expectativa positiva). Esse aumento de taxa não se confirma, tendo como valor a taxa efetiva de $8 \%$, fazendo nesse momento com que as expectativas dos agentes se alterem para pessimistas, esperando uma taxa futura de $7,5 \%$.

Para o quinto período, a taxa de juros apresenta uma inflexão e aumenta para $9 \%$. Como os agentes estão pessimistas em relação ao comportamento da economia, a expectativa de taxa de juros aumenta para apenas $8,5 \%$. No sexto 
período, a taxa de juros sobe para 10\% e os agentes continuam pessimistas, ajustam as suas expectativas para apenas $9,75 \%$. Por fim, no sétimo período a taxa de juros sobe para $11 \%$ e os agentes alteram suas expectativas novamente e se tornam otimistas, ajustando as suas expectativas para $11,5 \%$.

Os valores obtidos para $\Delta i$ evidenciam que o sinal dessa variável consegue identificar o comportamento dos agentes. Quando o sinal é positivo significa que os agentes estão otimistas em relação à economia, por sua vez, quando o sinal é negativo os agentes estão pessimistas em relação à economia. A magnitude de $\Delta i$ identifica o quanto o mercado está otimista ou pessimista. Portanto, a vantagem dessa variável é que, além de identificar se as expectativas dos agentes estão aumentando ou diminuindo, ela também consegue captar a intensidade das expectativas.

Quadro 1: Comportamento da variação dos juros.

\begin{tabular}{|r|r|r|r|}
\hline \multicolumn{1}{|c|}{ Período } & \multicolumn{1}{|c|}{ Juros } & Expectativa de Juros & $\boldsymbol{\Delta}_{\mathbf{i}}$ \\
\hline 1 & 11,00 & 11 & 0 \\
\hline 2 & 10,00 & 10,5 & 0,50 \\
\hline 3 & 9,00 & 9,5 & 0,50 \\
\hline 4 & 8,00 & 7,5 & $-0,50$ \\
\hline 5 & 9,00 & 8,5 & $-0,50$ \\
\hline 6 & 10,00 & 9,75 & $-0,25$ \\
\hline 7 & 11,00 & 11,5 & 0,50 \\
\hline
\end{tabular}

Fonte: Elaboração própria

\section{Resultados obtidos}

A presente seção irá apresentar os resultados obtidos, contudo, antes é necessário apresentar os dados utilizados. Os dados são de frequência diária e compreendem o período de o1/o1/2008 a 27/02/2015. Mais precisamente, as variáveis utilizadas foram: taxa de câmbio e juros $\left(\mathrm{S}_{\mathrm{t}} ; \mathrm{i}_{\mathrm{t}}\right)$; Expectativas de câmbio e juros um mês à frente $\left(\mathrm{S}_{\mathrm{t}, \mathrm{e}} ; \mathrm{i}_{\mathrm{t}, \mathrm{e}}\right)$. Essas variáveis são importantes indicadores macroeconômicos para os investidores alocarem seu capital em ativos nacionais. Desvio das expectativas de câmbio e juros um mês à frente $\left(\mathrm{S}_{\mathrm{t}}\right.$; $\left.\mathrm{i}_{\mathrm{t}}{ }_{\mathrm{t}}\right)$, por sua vez, essas duas variáveis captam a percepção de risco dos investidores. Índice setorial de energia elétrica (IEE); índice industrial (INDX); índice consumo (Icon); índice imobiliário (Imob); índice de materiais básico (Imat); índice de utilidade pública (Util); índice ibovespa (Ibov) e índice financeiro (IFNX). Esses são os índices setoriais do Ibovespa. Dummie cambial $\left(D_{e}\right)$, essa variável capta a desvalorização cambial e sua consequente evasão de divisas. O quadro 2 apresenta todas as variáveis que serão utilizadas no presente estudo e as fontes destas. 
Quadro 2: Variáveis utilizadas

\begin{tabular}{|c|c|c|c|}
\hline Símbolo & Descrição & Fonte & Unidade \\
\hline$S_{t}$ & Taxa de Câmbio & BCB-SGS* & $\mathrm{R} \$ / \mathrm{US}$ \\
\hline$i_{t}$ & Taxa de Juro & BCB-SGS & Percentual \\
\hline$s_{t, e}$ & Expectativa de Câmbio do mercado um mês a frente & BCB-SGS & RS/US\$ \\
\hline$s_{t}^{e}$ & Desvio da Taxa de Câmbio em relação as expectativas dos agentes & Notas do $\mathrm{BCB}$ & $\mathrm{R} \$$ /US\$ \\
\hline$D_{e}$ & Dummie Cambial & Notas do $\mathrm{BCB}$ & \\
\hline$i_{t}^{e}$ & Desvio da Taxa de Juros do Mercado um mês a frente & Notas do $\mathrm{BCB}$ & Percentual \\
\hline$i_{t, e}$ & Expectativa de Juros do mercado um mês a frente & BCB-SGS & Percentual \\
\hline iee $_{t}$ & Índice Energia Eletrica & Economática & Índice \\
\hline $\operatorname{ind} d x_{t}$ & Índice Industrial & Economática & Índice \\
\hline icont & Índice Consumo & Economática & Índice \\
\hline$i m o b t$ & Índice Imobiliário & Economática & Índice \\
\hline imatt & Índice de Materiais Básicos & Economática & Índice \\
\hline utilt & Índice de Utilidade Pública & Economática & Índice \\
\hline$i b o v t$ & Índice Ibovespa & Economática & Índice \\
\hline ifnxt & Índice Financeiro & Economática & Índice \\
\hline
\end{tabular}

Fonte: Elaboração própria

O Quadro 3 apresenta as estatísticas descritivas obtidas para os ativos analisados. Os resultados apresentados evidenciam que o Ibov e o INDX são os ativos que apresentam maior desvio-padrão, enquanto que IEE e Imat são os índices que apresentam menores desvios-padrões. Portanto, esses dois últimos são os ativos setoriais que apresentam menor dispersão nos retornos. Por sua vez, IEE, INDX e Imob apresentam curtose superior a três (apresenta distribuição leptocurtica, isto é, sua curva é mais achatada que a normal), e os valores encontrados para a assimetria mostram que Imat, IFNX e Icom apresentam assimetria positiva (valores estão concentrados à direita da média). $\mathrm{O}$ teste de Jarque-Bera indica que nenhum dos ativos analisados possui distribuição normal e a estatística de Dickey Fuller indica que o retorno de todos os ativos é estacionário (não existe raiz unitária nos retornos dos ativos). 
Quadro 3: Estatísticas Descritivas

\begin{tabular}{|c|c|c|c|c|c|c|c|c|}
\hline Ativo & Média & Mínimo & Máximo & Desvio padrão & Curtose & Assimetria & Jarque Bera & ADF \\
\hline iee. & 0,00 & $-0,09$ & 0,12 & 0,01 & 11,77 & $-0,02$ & \begin{tabular}{|r|}
5502,84 \\
\end{tabular} & $-19,8$ \\
\hline$i n d x_{t}$ & $1,0 E+04$ & $4,4 \mathrm{E}+03$ & $1,3 \mathrm{E}+04$ & $1,9 \mathrm{E}+03$ & 3,91 & $-1,17$ & 4147,45 & $-21,1$ \\
\hline icont & 1716,84 & 602,23 & 2860,00 & 609,89 & 1,76 & 0,01 & 4629,11 & $-20,5$ \\
\hline$i m o b t$ & 764,36 & 213,48 & 1143,00 & 180,78 & 3,59 & $-0,92$ & 3152,33 & $-21,0$ \\
\hline imatt $_{\text {imat }}$ & 1859,80 & 934,81 & 3156,57 & 401,89 & 2,91 & 0,44 & 1895,54 & $-20,1$ \\
\hline utilt & 2383,86 & 1162,60 & 3553,00 & 550,73 & 2,17 & $-0,19$ & 5160,87 & $-20,0$ \\
\hline$i b o v t$ & $5,7 \mathrm{E}+04$ & $2,9 \mathrm{E}+04$ & $7,4 \mathrm{E}+04$ & $8,5 \mathrm{E}+03$ & 2,91 & $-0,46$ & 3167,53 & $-20,0$ \\
\hline ifnxt & 3514,86 & 1325,48 & 5848,47 & 866,68 & 2,81 & 0,03 & 5364,81 & $-20,0$ \\
\hline$D_{e}$ & $-0,01$ & $-0,22$ & 0,12 & 0,04 & 6,07 & $-0,94$ & 5379,30 & $-7,4$ \\
\hline$i_{t}^{e}$ & 0,17 & $-0,65$ & 0,86 & 0,37 & 2,39 & $-0,31$ & $2,02 E+04$ & $-2,0$ \\
\hline$s_{t}^{e}$ & 0,22 & 0,00 & 1,00 & 0,42 & 2,77 & 1,33 & 1022,50 & $-12,2$ \\
\hline
\end{tabular}

Fonte: Elaboração própria

A Figura 1 (anexo) indica a ocorrência de três clusters de volatilidade nos ativos analisados: 1) em 2008, provocado pela crise do subprime; 2) no terceiro trimestre de 2011; e 3) a partir do terceiro trimestre de 2014. Esses resultados vão ao encontro da hipótese levantada de que existe transmissão de risco entre esses ativos, e que há também aumento da volatilidade em períodos de crise. A única exceção é IEE, cuja volatilidade apresenta uma instabilidade crescente a partir de 2012.

A Figura 2 (anexo) apresenta os valores encontrados para as expectativas dos juros e câmbio futuro. Os resultados consolidados na figura indicam que as expectativas de câmbio (gráfico da direita) estão sendo corrigidas rapidamente, enquanto que as expectativas em relação ao comportamento da taxa de juros (gráfico da esquerda) levam um período maior para serem corrigidas.

A explicação para tal ocorrência é que a taxa de câmbio é definida pela interação entre a oferta e a demanda, o desvio das expectativas em relação à taxa de câmbio é rapidamente corrigido. Esses resultados vão ao encontro da lei da arbitragem e a teoria de mercados eficientes, que argumentam que qualquer desvio entre o preço de mercado e o preço ótimo é rapidamente corrigido (Fama, 1970). Como uma proporção elevada dos ativos que compõem o mercado financeiro pertence a investidores estrangeiros, os desvios das expectativas de câmbio em relação à taxa de câmbio podem captar a percepção de risco dos investidores estrangeiros em relação à economia brasileira.

A taxa Selic não é definida apenas pelas forças do mercado. O Banco Central possui o monopólio da oferta de moeda e pode utilizar a política monetária para alcançar diferentes objetivos. A consequência disso é que pode existir uma discordância, no curto prazo, entre a taxa de juros definida pelo Banco Central e a taxa de juros esperada pelo mercado. No longo prazo, a lei da arbitragem garante a convergência entre a taxa de juros definida pelo Banco Central e as expectativas do 
mercado. Contudo, essa discordância entre a política realizada pelo Banco Central e a política ideal defendida pelo mercado leva a um aumento da incerteza. Por sua vez, esse aumento da incerteza em relação à condução da política monetária, acaba influenciando no comportamento dos ativos que compõem o mercado financeiro, aumentando a sua volatilidade, convergindo com os resultados encontrados por Forbes e Rigobon (2002) e Righi e Ceretta (2013), que apontam a incerteza como um dos motivos pela elevação da volatilidade.

Os critérios de informação de Akaike (AIC) e o critério bayesiano de Schwarz $(B I C)^{5}$ foram utilizados para identificar quantas defasagens deveriam ser utilizadas para estimar os modelos Arma, cujo resíduo foi utilizado para testar a presença de causalidade em variância. Os valores encontrados para o AIC e BIC indicam que o modelo Arma $(2,2)$ deve ser utilizado para todos os ativos. A única exceção é o Icon, que deve ser estimado através de um modelo Arma $(1,1)$.

Quadro 4: Critérios de informação de Akaike (AIC) e de Schwarz (BIC)

\begin{tabular}{|c|c|c|c|c|c|c|c|c|c|c|c|c|}
\hline \multirow[b]{2}{*}{$\operatorname{arma}(2,2)$} & \multirow[b]{2}{*}{ IEE } & AIC & -10.288 & -10.287 & -10.286 & -10.292 & -10.290 & -10.289 & -10.290 & -10.281 & $\mid-10.279$ & -10.274 \\
\hline & & BIC & -10.271 & -10.265 & -10.258 & -10.258 & -10.251 & -10.244 & -10.240 & -10.225 & -10.218 & -10.208 \\
\hline \multirow[b]{2}{*}{$\operatorname{arma}(1,1)$} & \multirow[b]{2}{*}{ INDX } & AIC & -9.3907 & -9.3913 & -9.3914 & -9.3951 & -9.3934 & -9.3918 & -9.3893 & -9.3806 & -9.3779 & -9.3793 \\
\hline & & $\mathrm{BIC}$ & -9.3741 & -9.3691 & -9.3637 & -9.3618 & -9.3545 & -9.3474 & -9.3394 & -9.3251 & -9.3169 & -9.3127 \\
\hline \multirow[b]{2}{*}{$\operatorname{arma}(2,2)$} & \multirow[b]{2}{*}{ ICON } & AIC & -9.9628 & -9.9634 & -9.9592 & -9.9648 & -9.9635 & -9.9589 & -9.9570 & -9.9481 & -9.9467 & -9.9454 \\
\hline & & $\mathrm{BIC}$ & -9.9461 & -9.9412 & -9.9314 & -9.9315 & -9.9247 & -9.9145 & -9.9071 & -9.8926 & -9.8856 & -9.8788 \\
\hline \multirow[b]{2}{*}{$\operatorname{arma}(2,2)$} & \multirow[b]{2}{*}{ IMOB } & AIC & -8.1654 & -8.1649 & -8.1454 & -8.1653 & -8.1460 & -8.1446 & -8.1420 & -8.1364 & -8.1333 & -8.1354 \\
\hline & & BIC & -8.1488 & -8.1427 & -8.1177 & -8.1320 & -8.1072 & -8.1002 & -8.0921 & -8.0809 & $\begin{array}{l}-8.0723 \\
\end{array}$ & -8.0688 \\
\hline \multirow[b]{2}{*}{$\operatorname{arma}(2,2)$} & \multirow[b]{2}{*}{ IMAT } & AIC & -8.4959 & -8.4960 & -8.4933 & -8.5014 & -8.4970 & -8.4959 & -8.4941 & -8.4858 & -8.4815 & -8.4812 \\
\hline & & $B I C$ & -8.4792 & -8.4738 & -8.4656 & -8.4681 & -8.4581 & -8.4515 & -8.4441 & -8.4303 & -8.4204 & -8.4146 \\
\hline \multirow[b]{2}{*}{$\operatorname{arma}(2,2)$} & \multirow[b]{2}{*}{ UTIL } & AIC & -9.8821 & -9.8803 & -9.8791 & -9.8866 & -9.8844 & -9.8833 & -9.8847 & -9.8718 & -9.8716 & -9.8670 \\
\hline & & $\mathrm{BIC}$ & -9.8655 & -9.8581 & -9.8513 & -9.8533 & -9.8455 & -9.8389 & -9.8348 & -9.8163 & -9.8106 & -9.8004 \\
\hline \multirow[b]{2}{*}{$\operatorname{arma}(2,2)$} & \multirow[b]{2}{*}{ IBOV } & AIC & -9.0185 & -9.0135 & -9.0125 & -9.0200 & -9.0178 & -9.0174 & -9.0147 & -9.0014 & -8.9992 & -9.0002 \\
\hline & & BIC & -9.0018 & -8.9913 & -8.9847 & -8.9867 & -8.9789 & -8.9730 & -8.9647 & -8.9459 & -8.9381 & -8.9336 \\
\hline \multirow[b]{2}{*}{$\operatorname{arma}(2,2)$} & \multirow[b]{2}{*}{ IFNX } & AIC & -8.6717 & -8.6704 & -8.6700 & -8.6865 & -8.6828 & -8.6886 & -8.6813 & -8.6556 & -8.6539 & -8.6598 \\
\hline & & $B I C$ & -8.6551 & -8.6482 & -8.6423 & -8.6532 & -8.6439 & -8.6442 & -8.6313 & -8.6001 & -8.5928 & -8.5931 \\
\hline
\end{tabular}

Fonte: Elaboração própria

Com a finalidade de observar a transmissão de volatilidade entre os ativos setoriais do Ibovespa, é realizado o teste de causalidade em variância e os resultados são apresentados no quadro 5. A interpretação do quadro ocorre de maneira temporal, o qual possui a relação de tempo defasada. Ademais, a interpretação é realizada através do teste $\mathrm{Q}$, representada pelos valores que, para

\footnotetext{
5 Os dois critérios apresentados utilizam da função verossimilhança como medida de ajustamento, contudo, definem valores críticos diferentes. Mais precisamente, o critério AIC busca minimizar a divergência entre a variável dependente e as variáveis exploratórias. Por sua vez, o critério BIC tenta maximizar a probabilidade da escolha do modelo verdadeiro. Os modelos com menor AIC e BIC são os considerados de melhor ajuste (BOZDOGAN, 1987).
} 
terem significância de pelo menos $95 \%$, eles precisam ser maiores que 1,64 (número referente à estatística $\mathrm{T}$ ), sendo marcados quando significantes com o “*” posteriormente ao seu número.

Ao observar o quadro 5, pode-se verificar que na defasagem de segunda ordem, definida pelos critérios de informação apresentados anteriormente, Ibov causa Icon, IEE e Util com intensidades diferentes, sendo elas, respectivamente, 5,58, 2,82 e 2,21. Esse raciocínio deve se estender por todas as linhas da figura. Dessa maneira, pode-se observar que Icon (observado na primeira defasagem, $t_{-1}$ ) causa apenas Util. Por sua vez, o índice de energia elétrica (IEE) causa: Icon, Imat e INDX com os valores de $2,76,1,76$ e 2,49 respectivamente. O IFNX causa apenas Imob com valor de 3,52. Imat causa Icon, IEE e Util. Imob causa apenas Util. Ademais, INDX causa apenas Imob e Util e por fim, Util causa apenas Icon e INDX.

O maior valor encontrado para essa transmissão é a causalidade entre Imat e Util que possui valor de 5,92. Uma das possíveis razões para haver uma causalidade 
Quadro 5: Valor encontrado para o teste de Causalidade em variância

\begin{tabular}{|c|c|c|c|c|c|}
\hline Discriminação & $t-1$ & $t-2$ & $t-3$ & $t-4$ & $t-5$ \\
\hline IBOV causa ICON & $2,59 *$ & $2,82^{*}$ & $3,61^{*}$ & $3,61^{*}$ & $3,62^{*}$ \\
\hline IBOV causa IEE & $2,04^{*}$ & $2,21^{*}$ & $2,95 *$ & $2,96 *$ & $3^{*}$ \\
\hline IBOV causa IFNX & $-0,39$ & $-0,18$ & $5,9^{*}$ & $5,89 *$ & $5,87^{*}$ \\
\hline IBOV causa IMAT & $-0,44$ & $-0,87$ & 0,71 & 0,87 & 0,87 \\
\hline IBOV causa IMOB & 0,63 & 0,88 & 2* & $2,11^{*}$ & $2,12^{*}$ \\
\hline IBOV causa INDX & $-0,19$ & $-0,35$ & 0,97 & 0,99 & 0,99 \\
\hline IBOV causa UTIL & $4,89 *$ & $5,58 *$ & $6,24 *$ & $6,19^{*}$ & $6,21^{*}$ \\
\hline ICON causa IBOV & $-0,24$ & $-0,67$ & 1,09 & 1,01 & 0,98 \\
\hline ICON causa IEE & 0,62 & 0,62 & $2,08^{*}$ & $2,07^{*}$ & $2,08^{*}$ \\
\hline ICON causa IFNX & $-0,15$ & $-0,43$ & $5,37 *$ & $5,3^{*}$ & $5,28^{*}$ \\
\hline ICON causa IMAT & $-0,43$ & $-0,78$ & 0,26 & 0,19 & 0,16 \\
\hline ICON causa IMOB & 1,31 & $2,51 *$ & $3,27^{*}$ & $3,17^{*}$ & $3,14^{*}$ \\
\hline ICON causa INDX & $-0,10$ & $-0,05$ & 1,04 & 1,03 & 1,04 \\
\hline ICON causa UTIL & $2,64^{*}$ & $2,88^{*}$ & $3,97 *$ & $3,85^{*}$ & $3,84^{*}$ \\
\hline IEE causa IBOV & 1,44 & 1,38 & $2,37^{*}$ & $2,36^{*}$ & $2,35 *$ \\
\hline IEE causa ICON & $2,54^{*}$ & $2,76^{*}$ & $3,32^{*}$ & $3,34^{*}$ & $3,36^{*}$ \\
\hline IEE causa IFNX & 0,20 & 0,18 & $4,33^{*}$ & $4,23 *$ & $4,21^{*}$ \\
\hline IEE causa IMAT & 1,23 & $1,76^{*}$ & $2,22^{*}$ & $2,18^{*}$ & $2,17^{*}$ \\
\hline IEE causa IMOB & $-0,26$ & $-0,19$ & 0,06 & 0,10 & 0,18 \\
\hline IEE causa INDX & $1,9^{*}$ & $2,49 *$ & $3,06^{*}$ & $3,01^{*}$ & $3,01^{*}$ \\
\hline IEE causa UTIL & $-0,16$ & $-0,53$ & 0,89 & 0,93 & 0,94 \\
\hline IFNX causa IBOV & $-0,48$ & $-0,92$ & 0,44 & 0,47 & 0,47 \\
\hline IFNX causa ICON & 0,09 & $-0,20$ & $-0,27$ & $-0,26$ & $-0,24$ \\
\hline IFNX causa IEE & $-0,29$ & $-0,61$ & $-0,37$ & $-0,36$ & $-0,34$ \\
\hline IFNX causa IIMOB & $2,75^{*}$ & $3,52^{*}$ & $3,69 *$ & $3,72^{*}$ & $3,74^{*}$ \\
\hline IFNX causa IMAT & $-0,29$ & $-0,33$ & 0,37 & 0,48 & 0,49 \\
\hline IFNX causa INDX & $-0,49$ & $-0,02$ & 0,23 & 0,23 & 0,23 \\
\hline IFNX causa UTIL & 0,43 & 0,18 & 0,42 & 0,42 & 0,42 \\
\hline IMAT causa IBOV & $-0,32$ & $-0,69$ & 0,99 & 0,96 & 0,93 \\
\hline IMAT causa ICON & $2,32^{*}$ & $2,5^{*}$ & $3,47^{*}$ & $3,46^{*}$ & $3,48^{*}$ \\
\hline IMAT causa IEE & $2,17^{*}$ & $2,62^{*}$ & $3,08^{*}$ & $3,09^{*}$ & $3,13^{*}$ \\
\hline IMAT causa IFNX & $-0,28$ & $-0,06$ & $4,87^{*}$ & $4,94^{*}$ & $4,92^{*}$ \\
\hline IMAT causa IMOB & $-0,01$ & 0,07 & 0,88 & 1,02 & 1,02 \\
\hline IMAT causa INDX & $-0,49$ & $-0,92$ & 0,68 & 0,70 & 0,70 \\
\hline IMAT causa UTIL & $5,02^{*}$ & $5,92^{*}$ & $6,22^{*}$ & $6,09^{*}$ & $6,09 *$ \\
\hline IMOB causa IBOV & $-0,38$ & $-0,43$ & 0,39 & 0,29 & 0,27 \\
\hline IMOB causa ICON & $-0,49$ & 0,00 & 0,32 & 0,32 & 0,33 \\
\hline IMOB causa IEE & 0,83 & 0,67 & 0,95 & 0,96 & 0,97 \\
\hline IMOB causa IFNX & 1,17 & 1,59 & $4,82^{*}$ & $4,72^{*}$ & $4,7^{*}$ \\
\hline IMOB causa IMAT & 0,33 & 0,26 & 0,58 & 0,48 & 0,46 \\
\hline IMOB causa INDX & 0,11 & $-0,13$ & 0,20 & 0,24 & 0,26 \\
\hline IMOB causa UTIL & $2,32^{*}$ & $2,43^{*}$ & $2,68^{*}$ & $2,57^{*}$ & $2,55^{*}$ \\
\hline INDX causa IBOV & $-0,45$ & $-0,89$ & 1,16 & 1,09 & 1,07 \\
\hline INDX causa ICON & 1,07 & 1,08 & $2,5^{*}$ & $2,4^{*}$ & $2,42^{*}$ \\
\hline INDX causa IEE & 1,46 & 1,52 & $2,57^{*}$ & $2,56^{*}$ & $2,6^{*}$ \\
\hline \begin{tabular}{|l} 
INDX causa IFNX \\
\end{tabular} & 0,12 & 0,37 & $6,77^{*}$ & $6,76^{*}$ & $6,74^{*}$ \\
\hline INDX causa IMAT & 0,23 & $-0,09$ & 1,25 & 1,21 & 1,19 \\
\hline INDX causa IMOB & 1,45 & $2,02^{*}$ & $3,01^{*}$ & $2,96^{*}$ & 2,93* \\
\hline INDX causa UTIL & $4,21^{*}$ & $4,78^{*}$ & $5,37^{*}$ & $5,24^{*}$ & $5,24^{*}$ \\
\hline UTIL causa IBOV & 1,32 & 1,25 & $2,48^{*}$ & $2,45 *$ & $2,43^{*}$ \\
\hline UTIL causa ICON & $2,56^{*}$ & $2,85^{*}$ & $3,67^{*}$ & $3,74^{*}$ & $3,77^{*}$ \\
\hline \begin{tabular}{|l|} 
UTIL causa IEE \\
\end{tabular} & $-0,42$ & $-0,87$ & 1,05 & 1,41 & 1,43 \\
\hline UTIL causa IFNX & $-0,12$ & 0,03 & $4,42^{*}$ & $4,34^{*}$ & $4,31^{*}$ \\
\hline UTIL causa IMAT & 1,09 & 1,42 & $2 *$ & $2,03^{*}$ & $2,03^{*}$ \\
\hline UTIL causa IMOB & $-0,35$ & $-0,57$ & $-0,01$ & 0,15 & 0,20 \\
\hline UTIL causa INDX & 1,58 & $2,05 *$ & $2,81^{*}$ & $2,83^{*}$ & $2,84^{*}$ \\
\hline
\end{tabular}

Fonte: Elaboração própria 
dessa magnitude entre e Imat e Util é o fato do Imat ser representado pelas empresas de fornecedores de materiais básicos e, dessa maneira, essas empresas são muito dependentes das empresas que compõem o índice de utilidade pública, relacionadas, por sua vez, ao fornecimento de energia, água e gás, principalmente. Dessa forma, haveria uma relação de causa e efeito de grande proporção. Por fim, um fato a se destacar nos resultados é que no momento que há aumento da incerteza, essa transmissão não ocorre para todos os ativos que compõem o mercado.

O teste de causalidade em variância é realizado novamente para o período entre o1 de janeiro de 2010 a 27 de fevereiro de 2015. A justificativa é a ocorrência da crise de 2008, que devido a sua magnitude pode ter enviesado os resultados obtidos, pelo aumento da volatilidade observado nesse período, como indica a literatura. Dessa forma, o quadro 6 apresenta os resultados obtidos por meio desse novo teste.

De maneira geral, nos resultados encontrados para o período após 2010, quadro 6, pode-se observar uma redução de causalidade entre os índices setoriais do Ibovespa. Assim, pode-se concluir que a nova realização do teste para o período posterior à crise melhorou a análise dos resultados, pois algumas causalidades encontradas anteriormente diminuíram sua expressão e/ou perderam confiança, haja vista a redução dos valores apresentados.

Mais precisamente, ao observar o quadro 6, verifica-se que INDX causa Icon; Icon causa INDX; INDX causa Imob; Imob causa INDX; Icon causa Imat; Icon causa Ibov; Ibov causa Icon; Imob causa Util; e por fim, Util causa Imob são rejeitadas. Esses resultados estão de acordo com a teoria, que argumenta que em períodos de incerteza, aumenta-se a volatilidade dos ativos e pode haver mudança no sentido da transmissão de risco. Os únicos ativos para os quais o teste de causalidade em variância para todo o período indicava a ausência de causalidade e passa a indicar a presença de causalidade após 2010 são: IEE causa Imob e Imob causa IEE, Ibov causa Imob, IFNX causa IEE, IFNX causa Util e Util e IFNX. A explicação é que, ao utilizar a segmentação correta da amostra, a causalidade setorial é melhor captada.

O quadro 7 mostra os valores encontrados para o modelo VAR para todo o período. O valor encontrado para a dummie que identifica três períodos - ou mais de desvalorização cambial é significativa para todos os ativos. As únicas exceções são Icon e Imat. Dada a elevada presença de investidores estrangeiros no mercado financeiro doméstico, esse resultado está de acordo com o esperado. Uma desvalorização cambial significa a evasão desses investidores, implicando em retornos menores para os ativos domésticos. O IFNX e o Imob são os índices que mais explicam o retorno dos demais índices. Uma variação do retorno do IFNX resulta em uma variação, em mesmo sentido, nos retornos do IEE, Icon, Imat, Util 
e Ibov. O retorno do Imob também apresenta uma relação positiva com INDX, Icon e Imob. 
Quadro 6: Valor encontrado para o teste de Causalidade em variância - após 2010

\begin{tabular}{|c|c|c|c|c|c|}
\hline Descriminação & $t-1$ & $t-2$ & $t-3$ & $t-4$ & $t-5$ \\
\hline IEE causa INDX & $1,66096350600867^{*}$ & $2,12175757010408^{*}$ & $2,80051952275373^{*}$ & $2,74583806250481^{*}$ & $2,74472441816309 *$ \\
\hline INDX causa IEE & $2,37582458564214^{*}$ & 3,3648838885593* & $3,36336465214655 *$ & $3,35217765401586^{*}$ & $3,36545576442907 *$ \\
\hline IEE causa ICON & 1,05 & 1,27 & \begin{tabular}{|l|l|}
7 & 1,38 \\
\end{tabular} & 1,46 & \begin{tabular}{|r|}
6 \\
1,45
\end{tabular} \\
\hline ICON causa IEE & 1,37 & $2,51159692220751^{*}$ & $2,74369276279053^{*}$ & $2,72777540969692^{*}$ & $2,7277736566912^{*}$ \\
\hline IEE causa IMOB & 0,66 & 1,03 & \begin{tabular}{|r|r}
3 & 0,79 \\
\end{tabular} & 0,95 & \begin{tabular}{|r|r}
5,97 \\
\end{tabular} \\
\hline IMOB causa IEE & $1,66639096908186 *$ & $1,98004212057787^{*}$ & $1,71824794288757^{*}$ & $1,74483157221736^{*}$ & $1,74574809090495 *$ \\
\hline IEE causa IMAT & 4,0114360662913* & 5,37437690973709* & 5,13880561675996* & $5,04302170202951^{*}$ & 5,01798334151139* \\
\hline IMAT causa IEE & 3,01993383989979* & $4,39611543047841^{*}$ & $4,12599169040876^{*}$ & $4,13902568326173^{*}$ & $4,14847622795289 *$ \\
\hline IEE causa UTIL & $-0,46$ & $-0,54$ & \begin{tabular}{|l|l}
4 & $-0,03$ \\
\end{tabular} & 0,11 & 0,09 \\
\hline UTIL causa IEE & $-0,48$ & $-0,09$ & 0,38 & 0,71 & 0,71 \\
\hline IEE causa IBOV & $-0,11$ & $-0,50$ & 0,00 & $-0,09$ & $-0,09$ \\
\hline IBOV causa IEE & $3,18725783885308^{*}$ & $4,4332461294925^{*}$ & $4,24695452147112^{*}$ & $4,24619509166496^{*}$ & $4,25138621429843^{*}$ \\
\hline IEE causa IFNX & $1,85092965660368^{*}$ & $2,61925352308634^{*}$ & $4,29289034755883^{*}$ & $4,42852738643915^{*}$ & 4,45989353764674* \\
\hline IFNX causa IEE & $-0,07$ & 0,34 & 0,15 & 0,16 & 0,16 \\
\hline INDX causa ICON & $-0,09$ & 0,17 & 0,65 & 0,58 & 0,57 \\
\hline ICON causa INDX & $-0,04$ & 0,04 & 1,12 & 1,12 & 1,12 \\
\hline INDX causa IMOB & $-0,11$ & 0,30 & 0,12 & 0,22 & 0,22 \\
\hline IMOB causa INDX & 0,12 & 0,07 & 0,50 & 0,51 & 0,54 \\
\hline INDX causa IMAT & $-0,24$ & $-0,63$ & $-0,42$ & $-0,48$ & $-0,49$ \\
\hline IMAT causa INDX & $-0,49$ & $-0,95$ & 0,69 & 0,71 & 0,71 \\
\hline INDX causa UTIL & $2,08219753327318^{*}$ & $2,56247755714082^{*}$ & $2,67573497488923^{*}$ & $2,5742203890762^{*}$ & $2,55666950160262^{*}$ \\
\hline UTIL causa INDX & 1,39 & $1,82081282997666^{*}$ & $2,75982779425499 *$ & $2,76491526095499 *$ & $2,77081938684532^{*}$ \\
\hline INDX causa IBOV & $-0,01$ & $-0,17$ & 0,63 & 0,63 & 0,62 \\
\hline IBOV causa INDX & $-0,12$ & $-0,10$ & 1,34 & 1,36 & 1,36 \\
\hline INDX causa IFNX & $-0,42$ & 0,00 & $2,54170105031575^{*}$ & $2,69982774241113^{*}$ & $2,72183141821736^{*}$ \\
\hline IFNX causa INDX & $-0,49$ & 0,33 & 0,72 & 0,71 & 0,71 \\
\hline ICON causa IMOB & $-0,18$ & 1,19 & 0,95 & 0,92 & 0,92 \\
\hline IMOB causa ICON & $-0,33$ & $1,68287668753648^{*}$ & 1,51 & 1,53 & 1,54 \\
\hline ICON causa IMAT & 1,30 & 1,52 & 1,60 & 1,50 & 1,49 \\
\hline IMAT causa ICON & 0,91 & 1,18 & 1,50 & 1,56 & 1,56 \\
\hline ICON causa UTIL & 0,92 & 1,19 & $1,68342144515226^{*}$ & 1,58 & 1,56 \\
\hline UTIL causa ICON & 0,97 & 1,22 & 1,57 & $1,67345098459733^{*}$ & $1,67995116092195^{*}$ \\
\hline ICON causa IBOV & $-0,40$ & $-0,81$ & $-0,09$ & $-0,09$ & $-0,12$ \\
\hline IBOV causa ICON & 0,84 & 0,90 & 1,16 & 1,21 & 1,21 \\
\hline ICON causa IFNX & $-0,35$ & $-0,46$ & $1,66380863971062^{*}$ & $1,88494721633708^{*}$ & $1,88133509746516^{*}$ \\
\hline IFNX causa ICON & $-0,45$ & $-0,93$ & $-1,08$ & $-1,07$ & $-1,07$ \\
\hline IMOB causa IMAT & $-0,47$ & $-0,79$ & $-0,87$ & $-0,95$ & $-0,97$ \\
\hline IMAT causa IMOB & 0,65 & 0,95 & 0,73 & 0,00 & 1,01 \\
\hline IMOB causa UTIL & 0,94 & 0,80 & 0,73 & 0,65 & 0,63 \\
\hline UTIL causa IMOB & 0,88 & 0,97 & 0,77 & 1,04 & 1,05 \\
\hline IMOB causa IBOV & 0,75 & $1,87843484774019 *$ & $1,97919883216583^{*}$ & $1,99166785607416^{*}$ & $1,99199974275354^{*}$ \\
\hline IBOV causa IMOB & 0,72 & 1,27 & 1,09 & 1,40 & 1,45 \\
\hline IMOB causa IFNX & $-0,37$ & 0,55 & 1,49 & $1,79458717668152^{*}$ & $1,79328973936169 *$ \\
\hline IFNX causa IIMOB & $\begin{array}{r}-0,47 \\
\end{array}$ & 0,22 & 0,01 & 0,14 & 0,24 \\
\hline IMAT causa UTIL & $2,81801892563932^{*}$ & $3,6095359254743^{*}$ & $3,56850499826851^{*}$ & $3,48334539951412^{*}$ & $3,46185220863679^{*}$ \\
\hline UTIL causa IMAT & 3,69072699591924* & 4,79853778912822* & $4,71399124331542^{*}$ & $4,68831427790317^{*}$ & $4,6958388659624^{*}$ \\
\hline IMAT causa IBOV & \begin{tabular}{r|}
$-0,24$ \\
\end{tabular} & $-0,21$ & 0,46 & 0,40 & $\begin{array}{r}0,38 \\
\end{array}$ \\
\hline IBOV causa IMAT & 0,87 & 0,94 & 1,25 & 1,35 & 1,38 \\
\hline IMAT causa IFNX & $-0,25$ & 0,58 & $2,57016922778333^{*}$ & $2,56732851753672^{*}$ & $2,56672899723204^{*}$ \\
\hline IFNX causa IMAT & 0,02 & 0,53 & 0,52 & 0,58 & 0,61 \\
\hline UTIL causa IBOV & $-0,15$ & $-0,57$ & 0,19 & 0,10 & 0,09 \\
\hline IBOV causa UTIL & $2,64393012100643^{*}$ & $3,28271439743382^{*}$ & $3,44450318844758^{*}$ & $3,43231689340957^{*}$ & $3,43227082370951^{*}$ \\
\hline UTIL causa IFNX & 1,14 & $1,8018984462188^{*}$ & $3,63087266636468^{*}$ & $3,73253306570249 *$ & $3,729476279803^{*}$ \\
\hline IFNX causa UTIL & $-0,31$ & $-0,42$ & $-0,42$ & $-0,42$ & $-0,42$ \\
\hline IBOV causa IFNX & 0,00 & 0,64 & $2,70542759174595^{*}$ & $2,94288718865959 *$ & $2,94304589559978^{*}$ \\
\hline IFNX causa IBOV & 0,29 & $-0,03$ & 0,48 & 0,56 & 0,56 \\
\hline
\end{tabular}

Fonte: Elaboração própria 
Quadro 7: Modelo VAR, com expectativas e variável binária para o câmbio - todo o período $^{6}$

\begin{tabular}{|c|c|c|c|c|c|c|c|c|c|c|c|c|c|}
\hline Ativo & discriminação & Constante & iee & ind $x$ & icon & imob & imat & util & ibovespa & ifnx & Dcambio & Djuro & DI \\
\hline \multirow[t]{2}{*}{ iee } & Estimativa & $0,000617^{* *}$ & $-0,02$ & 0 & 0,01 & $-0,01$ & 0 & 0,08 & $-0,18$ & $0,11^{* *}$ & $0,01^{* *}$ & $-0,00167$ & $-0,00112$ \\
\hline & Erro padrão & 0,000358 & 0,08 & 0,1 & 0,07 & 0,02 & 0,06 & 0,07 & 0,07 & 0,03 & 0,01 & 0,000731 & 0,000796 \\
\hline \multirow[t]{2}{*}{ ind $x$} & Estimativa & $0,000358^{* *}$ & 0,08 & 0,1 & \multicolumn{2}{|c|}{$0,070,02 * *$} & 0,06 & 0,07 & 0,07 & \multicolumn{2}{|c|}{$0,030,01^{* *}$} & 0,000731 & 0,000796 \\
\hline & Erro padrão & 0,000474 & 0,11 & 0,13 & 0,09 & 0,03 & 0,07 & 0,09 & 0,1 & 0,05 & 0,01 & 0,000967 & 0,00105 \\
\hline \multirow[t]{2}{*}{ icon } & Estimativa & $0,00079 * *$ & $-0,09$ & 0,21 & \multicolumn{2}{|c|}{$-0,110,06^{* *}$} & $-0,01$ & $-0,06$ & \multicolumn{2}{|c|}{$-0,140,05^{* *}$} & 0,02 & $-0,00264$ & $-0,00132$ \\
\hline & Erro padrão & 0,000401 & 0,09 & 0,11 & 0,07 & 0,03 & 0,06 & 0,08 & 0,08 & 0,04 & 0,01 & 0,000819 & 0,000891 \\
\hline \multirow[t]{2}{*}{ imob } & Estimativa & 0,000474 & 0,11 & 0,13 & \multicolumn{2}{|c|}{$0,090,03^{* *}$} & 0,07 & 0,09 & 0,1 & \multicolumn{2}{|c|}{$0,050,01^{* *}$} & 0,000967 & 0,00105 \\
\hline & Erro padrão & 0,000669 & 0,15 & 0,18 & 0,12 & 0,05 & 0,1 & 0,13 & 0,14 & 0,06 & 0,01 & 0,00136 & 0,00149 \\
\hline \multirow[t]{2}{*}{ imat } & Estimativa & 0,00107 & $-0,03$ & $0,06^{* *}$ & 0,01 & 0,07 & $-0,02$ & $-0,06$ & $-0,18$ & \multicolumn{2}{|c|}{$0,080,01^{* *}$} & $-0,00234$ & $-0,00135$ \\
\hline & Erro padrão & 0,000606 & 0,14 & 0,17 & 0,11 & 0,04 & 0,09 & 0,12 & 0,13 & 0,06 & 0,01 & 0,00124 & 0,00135 \\
\hline \multirow[t]{2}{*}{ util } & Estimativa & $0,000401^{* *}$ & 0,09 & 0,11 & 0,07 & 0,03 & 0,06 & 0,08 & \multicolumn{2}{|c|}{$0,080,04^{* *}$} & $0,01^{* *}$ & 0,000819 & 0,000891 \\
\hline & Erro padrão & 0,000402 & 0,09 & 0,11 & 0,08 & 0,03 & 0,06 & 0,08 & 0,08 & 0,04 & 0,01 & 0,000821 & 0,000894 \\
\hline \multirow[t]{2}{*}{ ibovespa } & Estimativa & 0,000633 & 0,02 & 0,25 & $-0,02$ & 0,16 & $-0,1$ & $-0,12$ & \multicolumn{2}{|c|}{$-0,270,08^{* *}$} & $0,03^{* *}$ & $-0,00349$ & $-0,00192$ \\
\hline & Erro padrão & 0,000523 & 0,12 & 0,14 & 0,1 & 0,04 & 0,08 & 0,1 & \multicolumn{2}{|c|}{$0,11 \quad 0$} & 0,01 & 0,00107 & 0,00116 \\
\hline \multirow[t]{2}{*}{ ifnx } & Estimativa & 0,000669 & 0,15 & 0,18 & 0,12 & 0,05 & 0,1 & 0,13 & \multicolumn{2}{|c|}{$0,140,06^{* *}$} & 0,01 & 0,00136 & 0,00149 \\
\hline & Erro padrão & 0,000576 & 0,13 & 0,16 & 0,11 & 0,04 & 0,09 & 0,11 & 0,12 & 0,05 & 0,01 & 0,00118 & 0,00128 \\
\hline \multirow[t]{2}{*}{ Dcambio } & Estimativa & 0,000515 & $-0,13$ & 0,29 & \multicolumn{2}{|c|}{$-0,190,04^{* *}$} & 0,02 & $-0,09$ & $-0,11$ & \multicolumn{2}{|c|}{$0,040,02^{* *}$} & $-0,00274$ & $-0,00156$ \\
\hline & Estimativa & 0,000678 & 0,15 & 0,18 & 0,13 & 0,05 & 0,11 & 0,13 & 0,14 & 0,06 & 0,01 & 0,00138 & 0,00151 \\
\hline \multirow[t]{2}{*}{ Djuro } & Erro padrão & 0,000606 & $0,14 * *$ & 0,17 & 0,11 & 0,04 & 0,09 & 0,12 & 0,13 & 0,06 & 0,01 & 0,00124 & 0,00135 \\
\hline & Estimativa & 0,00225 & 0,51 & 0,61 & 0,42 & 0,15 & 0,35 & 0,44 & 0,47 & 0,21 & 0,04 & 0,0046 & 0,00501 \\
\hline \multirow[t]{2}{*}{ DI } & Erro padrão & $0,000735^{* *}$ & $-0,02$ & $-0,02 * *$ & 0,01 & 0,01 & 0,01 & \multicolumn{2}{|c|}{$0,08-0,23^{* *}$} & 0,13 & 0,02 & $-0,00155^{*}=$ & $-0,0016$ \\
\hline & Erro padrão & 0,00746 & 1,68 & 2,03 & 1,39 & 0,51 & 1,16 & 1,45 & 1,54 & 0,71 & 0,14 & $\begin{array}{l}0,0152 \\
\end{array}$ & 0,0166 \\
\hline
\end{tabular}

Fonte: Elaboração própria

Para evitar o viés da crise do subprime, um modelo VAR é estimado para o período após 2010, o quadro 8 apresenta os valores encontrados. A comparação entre os valores consolidados no quadro 7 e 8 indicam que a variável binária que identifica a ocorrência de três ou mais períodos de desvalorização cambial permanece significativa apenas para Imat e Ibov. O IFXN passa a explicar os retornos do Imat, Icon, De e Djuros, porém, deixa de explicar os retornos do INDX, Ibov. O Imob passa a explicar o INDX, Imat, Ibov, IFNX e De; e deixa de explicar os retornos do Imob, IEE e Util. O Icon passa a explicar o Imob, Imat, IFNX, INDX, Ibov e Djuro. O índice Util passa a explicar IEE, INDX, Icon, Imat, Util, Ibov e o Juros. Todavia, Util deixa de explicar o Dcâmbio e o IFNX. O desvio das expectativas do câmbio passa a explicar Util e Ibov e o desvio das expectativas de juro passa a explicar o IFNX.

${ }^{6} \mathrm{O}$ símbolo de asteriscos $\left({ }^{* * *}\right),\left({ }^{* *}\right)$ e $\left({ }^{*}\right)$, nos quadros 7,8 e 9 , indica que a variável é estatisticamente significante a nível de $1 \%, 5 \%$ e $10 \%$ respectivamente. 
Quadro 8: Modelo VAR, com expectativas e variável binária para o câmbio - período pós 2010

\begin{tabular}{|c|c|c|c|c|c|c|c|c|c|c|c|c|c|}
\hline Ativo & discriminação & Constante & iee & ind $x$ & icon & imob & imat & util & ibovespa & ifnx & Dcambio & Djuro & DI \\
\hline \multirow[t]{2}{*}{ iee } & Estimativa & 0,00028 & 0,02 & $-0,07$ & 0,04 & 0,00042 & $-0,01^{*}$ & 0,07 & $-0,07^{*}$ & 0,06 & 0,01 & $-0,00047$ & $-0,00074$ \\
\hline & Erro padrão & 0,00039 & 0,09 & 0,12 & 0,09 & 0,03 & 0,06 & 0,08 & 0,08 & 0,04 & 0,01 & 0,00084 & 0,00076 \\
\hline \multirow[t]{2}{*}{ ind $x$} & Estimativa & $0,00063^{* * *}$ & $-0,06$ & $-0,19$ & $0,08^{* * *}$ & $0,09 * *$ & $0,05^{*}$ & $-0,02$ & $-0,02^{*}$ & 0,01 & 0 & $-0,0013$ & $-0,0012$ \\
\hline & Erro padrão & 0,00044 & 0,1 & 0,14 & 0,1 & 0,04 & 0,06 & 0,09 & 0,09 & 0,05 & 0,01 & 0,00095 & 0,00085 \\
\hline \multirow[t]{2}{*}{ icon } & Estimativa & 0,001 & $-0,08$ & $-0,25^{*}$ & 0,18 & 0,1 & $0,04^{*}$ & 0,03 & $-0,07^{*}$ & $0,03^{*}$ & 0 & $-0,0013$ & $-0,0012$ \\
\hline & Erro padrão & 0,00039 & 0,09 & 0,12 & 0,09 & 0,03 & 0,06 & 0,08 & 0,08 & 0,04 & 0,01 & 0,00084 & 0,00075 \\
\hline \multirow[t]{2}{*}{ imob } & Estimativa & 0,00017 & $-0,21$ & $-0,25$ & $0,13^{*}$ & 0,13 & $-0,06^{*}$ & 0,12 & $0,03^{*}$ & 0,1 & 0 & $-0,00058$ & $-0,0019$ \\
\hline & Erro padrão & 0,00064 & 0,15 & 0,2 & 0,15 & 0,06 & 0,09 & 0,13 & 0,13 & 0,07 & 0,01 & 0,0014 & 0,0012 \\
\hline \multirow[t]{2}{*}{ imat } & Estimativa & 0,00016 & 0,03 & 0,01 & $-0,09$ & 0,02 & $0,06^{*}$ & $-0,14$ & $0,05^{*}$ & $0,08^{*}$ & 0,00 & $-0,0009$ & $-0,0015$ \\
\hline & Erro padrão & 0,0006 & 0,14 & 0,19 & 0,14 & 0,05 & 0,09 & 0,12 & \begin{tabular}{|r|}
0,12 \\
\end{tabular} & 0,07 & 0,01 & 0,0013 & 0,0012 \\
\hline \multirow[t]{2}{*}{ util } & Estimativa & $0,0006^{* *}$ & 0 & $-0,06$ & 0,03 & 0 & $-0,02^{*}$ & 0,08 & $-0,1^{*}$ & 0,08 & 0,01 & $-0,00089$ & $-0,0012^{* *}$ \\
\hline & Erro padrão & 0,00043 & 0,1 & 0,14 & 0,1 & 0,04 & 0,06 & 0,09 & 0,09 & 0,05 & 0,01 & 0,00094 & 0,00084 \\
\hline \multirow[t]{2}{*}{ ibovespa } & Estimativa & 0,0003 & $-0,03$ & $-0,04$ & 0,02 & $0,04 *$ & $-0,01 *$ & $-0,04^{*}$ & $0,02 *$ & 0,1 & 0,01 & $-0,0011$ & $-0,0012$ \\
\hline & Erro padrão & 0,00052 & 0,12 & 0,16 & 0,12 & 0,05 & 0,08 & 0,1 & 0,11 & 0,06 & 0,01 & 0,0011 & 0,001 \\
\hline \multirow[t]{2}{*}{ ifnx } & Estimativa & 0,00063 & $-0,09$ & $-0,08$ & 0,12 & 0,02 & $0,01^{*}$ & 0,01 & $0,06^{*}$ & $-0,05$ & 0,02 & $-0,00068$ & $-0,00066$ \\
\hline & Erro padrão & 0,00054 & 0,12 & 0,17 & 0,12 & 0,05 & 0,08 & 0,11 & 0,11 & 0,06 & 0,01 & 0,0012 & 0,001 \\
\hline \multirow[t]{2}{*}{ Dcambio } & Estimativa & $-0,00059^{* *}$ & 0,09 & 0,19 & $-0,2^{* * *}$ & 0,02 & $-0,07^{*}$ & $-0,1$ & $0,18^{*}$ & $0,03^{*}$ & 0,9 & 0,0013 & $-0,00037$ \\
\hline & Estimativa & $\begin{array}{r}0,0006 \\
\end{array}$ & 0,14 & 0,19 & 0,14 & 0,05 & 0,09 & 0,12 & 0,13 & 0,07 & 0,01 & 0,0013 & 0,0012 \\
\hline \multirow[t]{2}{*}{ Djuro } & Erro padrão & 0,0026 & $-0,15$ & 0,8 & $-0,02$ & $-0,26^{*}$ & $-0,37^{*}$ & $-0,07$ & $0,74^{*}$ & $-0,74^{*}$ & 0,01 & 0,99 & $-0,0017$ \\
\hline & Estimativa & 0,0021 & 0,48 & 0,66 & 0,48 & 0,18 & 0,3 & 0,42 & 0,43 & 0,23 & 0,04 & 0,0045 & 0,004 \\
\hline \multirow[t]{2}{*}{$\mathrm{DI}$} & Erro padrão & 0,064 & 3,35 & 0,95 & 0,17 & $-0,13$ & $0,46^{*}$ & $-3,48$ & $-0,09^{*}$ & 0,1 & $-0,53^{*}$ & $-0,028$ & 0,72 \\
\hline & Erro padrão & 0,01 & 2,38 & 3,29 & 2,4 & 0,91 & 1,51 & 2,09 & 2,16 & 1,14 & 0,22 & 0,022 & 0,02 \\
\hline
\end{tabular}

Fonte: Elaboração própria

O teste de Ljung Box foi utilizado para identificar se os resíduos dos modelos VAR estimados são estacionários. Os resultados encontrados, Quadro 9, indicam que para o modelo sem expectativas, nenhum dos resíduos é estacionário. $\mathrm{O}$ resultado encontrado para o teste de Ljung Box para o modelo com expectativas estimado para todo o período indica que apenas os resíduos gerados pelo modelo VAR para INDX, Icon e Ibov são estacionários. Por outro lado, para o período após 2010, o teste de Ljung Box indica que todos os resíduos são estacionários. Portanto, o modelo VAR estimado para o período após 2010 possui um bom poder de explicação para todos os índices considerados.

Quadro 9: Resultados encontrados para o teste de Ljung Box

\begin{tabular}{|l|r|r|r|r|}
\hline \multicolumn{1}{|c|}{ Ativo } & Período todo & após 2010 & Expec. Período todo & Expec. após 2010 \\
\hline ie $\boldsymbol{~}_{t}$ & 26,66 & 15,23 & 34,28 & $24,45^{*}$ \\
\hline in $d x_{t}$ & 33,57 & 23,35 & $32,25^{*}$ & $28,05^{*}$ \\
\hline icont & 33,72 & 22,8 & $31,25^{*}$ & $28,05^{*}$ \\
\hline imobt & 33,80 & 22,69 & 26,49 & $29,76^{*}$ \\
\hline imat $t$ & 33,90 & 22,84 & 33,19 & $27,39^{*}$ \\
\hline utilt & 33,81 & 22,65 & 36,52 & $24,73^{*}$ \\
\hline ibovt & 32,22 & 23,29 & $32,35^{*}$ & $25,99^{*}$ \\
\hline ifnxt & 33,82 & 23,12 & 27,73 & $27,62^{*}$ \\
\hline
\end{tabular}

Fonte: Elaboração própria 


\section{Considerações finais}

O presente estudo procurou avaliar a transmissão de volatilidade entre os índices setoriais do Ibovespa. Para tanto, utilizou-se do teste de causalidade na variância, proposto por Cheung e Ng (1996). A partir dos resultados coletados e apresentados no decorrer do trabalho, pode-se concluir, com algumas exceções, que a maioria dos setores do Ibovespa pode inferir causalidade em um ou mais setores, e que essa relação de causalidade ocorre primordialmente de maneira bidirecional, embora também ocorra de maneira unidirecional. Ademais, foi encontrado que a temporalidade mais coerente para análise seria a $\mathrm{T} \pm 2$ (menos para os dados de Icon, para os quais a temporalidade mais adequada é $\mathrm{T} \pm 1$ ). Portanto, sendo os dados diários, foi possível afirmar que os índices interagem de maneira geral com uma defasagem de 2 dias.

As variáveis de expectativa utilizadas no modelo provaram ser úteis haja vista a influência da taxa de juros e do câmbio em economias emergentes. Pode-se observar que, a partir de sua utilização, quando as expectativas dos agentes divergem da realidade, ocorre uma elevação da incerteza, e que ela pode se tornar um contágio financeiro, transmitindo essa volatilidade entre os setores.

A realização de novos testes com dados posteriores aos de 2010 foi de fundamental importância, uma vez que após a segmentação das análises foi possível observar que a transmissão de risco diminui, demonstrando que os resultados poderiam estar enviesados pela elevada magnitude da crise de 2008 que, sistematicamente, elevou o risco global. Contudo, a análise separada trouxe maior fidedignidade à causalidade encontrada entre os índices. Dessa forma, foi possível ratificar a teoria que em períodos de incerteza existe um aumento da volatilidade geral do mercado, e que esses movimentos podem ser explicados como sendo a causa e/ou o efeito de um movimento em outro mercado.

O trabalho evidenciou a necessidade de aprofundamento no que se refere à transmissão de risco entre os mercados, considerando sua capacidade de explicação e aplicabilidade. Sua utilização pode vir desde algo mais aplicável e quântico, como controles de riscos para gestores de carteiras de investimento, como também, ser uma ferramenta de maior compreensão das relações intersetoriais existentes, auxiliando em tomadas de decisões macroeconômicas.

Referências bibliográficas

ALMEIDA, W. S. The recent evolution of the financial system: the brazilian case. The George Washington University, Institute of Brazilian Business and Public Managemnt Issues - IBI - 200o. 
BACA, S.; GARBE, B.; WEISS, R. The rise of sector effects in major equity markets. Financial Analysts Journal - Association for Investment Management and Research, set./ out. 2000.

BHAR, Hamori. S. Causality in variance and the type of traders in crude oil futures.

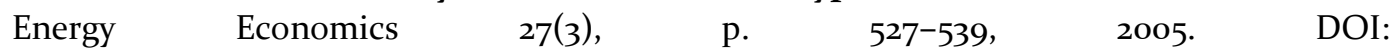
https://doi.org/10.1016/j.eneco.2004.12.003

BOLLERSLEV, T. Generalized autoregressive conditional heterocedasticity. Journal of Econometrics 31, p. 307-327, 1986. DOI: https://doi.org/10.1016/0304-4076(86)90063-1

BOZDOGAN, H. Model selection and Akaike's information criterion (AIC): The general theory and its analytical extensions. Psychometrica, n.52, p.345-370, 1987.

BRUTTI R. M.; CERETTA, P. S. EFEITO DA CRISE DE 2007/2008 NA TRANSMISSÃO INTERNACIONAL DE VOLATILIDADE NO MERCADO DE CAPITAIS BRASILEIRO. 2011. 1:10 f. (Escola de Administração) - Universidade Federal do Rio Grande do Sul, Rio Grande do Sul, 2013.

CAPORALE, G. M.; Pittis, N.; Spagnolo, N. Testing for causalityin-variance: an application to the east asian markets. International Journal of Finance \& Economics, 7(3), p. 235245, 2002. DOI: https://doi.org/10.1002/ijfe.185

CAPORIN, M.; MCALEER, M. Do We Really Need Both BEKK and DCC? A Tale of Two Multivariate GARCH Models. Working Paper, 2010.

CHEUNG, Y.W.; L. K. Ng. A causality-in-variance test and its application to financial market prices. Journal of Econometrics 72(1), p. 33-48, 1996. DOI: https://doi.org/10.1016/0304-4076(94)01714-X

CINTRA, M. A. M. A montagem de um novo regime monetário financeiro nos Estados Unidos: 1982-94. São Paulo: FUNDAP, 1998.

COPELAND, T. E.; WESTON, J. F.; SHASTRI, K. Financial Theory and Corporate Policy. New York: Pearson Education, 1998.

ENGLE, R. F. Autoregressive conditional heteroscedasticity with estimates of the variance of United Kingdom inflation. Econometrica: Journal of the Econometric Society, p. 987-1007, 1982. DOI: https://doi.org/10.2307/1912773

FAMA, E. The Behavior of Stock Market Prices. Journal of Business 38, p.34-105, 1965. DOI: https://doi.org/10.1086/294743 
FAMA, E. F. Efficient capital markets: A review of theory and empirical work*. The journal of Finance 25(2), p. 383-417, 1970. DOI: https://doi.org/10.2307/2325486

FORBES, K. J.; Rigobon, R. No contagion, only interdependence: measuring stock market comovements. The journal of Finance 57(5), p. 2223-2261, 2002. DOI: https://doi.org/10.1111/0022-1082.00494

GRANGER, C. W; Huangb, B.N.; Yang, C.W. A bivariate causality between stock prices and exchange rates: evidence from recent asian. The Quarterly Review of Economics and Finance 40(3), p. 337-354, 200o. DOI: https://doi.org/10.1016/S1062-9769(00)00042-9

GOODHART, C. A. E.; O'HARA, M. High frequency data in financial markets: Issues and applications. Journal of Empirical Finance, 4. p. 73-114, 1997. DOI: https://doi.org/10.1016/Sog27-5398(97)00003-0

HONG, Y. A test for volatility spillover with application to exchange rates. Journal of Econometrics 103(1), p. 183-224, 2001. DOI: https://doi.org/10.1016/So3044076(01)00043-4

HU, J. W.S.; Chen, M. Y.; Fok, R. C.; Huang, B. N. Causality in volatility and volatility spillover effects between us, japan and four equity markets in the south china growth triangular. Journal of International Financial Markets, Institutions and Money 7(4), p. 351-367, 1997. DOI: https://doi.org/10.1016/S1042-4431(97)00027-9

LO, Andrew; MACKINLAY, A. Craig. Stock Market Prices Do Not Follow Random Walks: Evidence from a Simple Specification Test. 2015. 44-66 p. Oxford University Press (Science Economics) - University of Oxford, Oxford, 2015.

PANTELIDIS, T.; Pittis, N. Testing for granger causality in variance in the presence of causality in mean. Economics Letters 85(2), p. 201-207, 2004. DOI: https://doi.org/10.1016/j.econlet.2004.04.006

PHYLAKTIS, K.; Xia, L.; Kolb, R. W. Equity market contagion and co-movement: Industry level evidence. Sovereign Debt: From Safety to Default, p. 369-378, 2011.

RIGOBON, R. International Financial Contagion: Theory and Evidence in Evolution. Massachusetts Institute of Technology: Sloan School of Management, 2002.

RIGHI, M. B.; Ceretta, P. S. Efeito da crise de 2007/2008 na transmissão internacional de volatilidade no mercado de capitais brasileiro. REAd-Revista Eletrônica de Administração 75(2), p. 384-400, 2013. DOI: https://doi.org/10.1590/S141323112013000200005 
SHARPE, W. F.; ALEXANDER, Gordon J.; BAILEY, Jeffery V. Investments. New Jersey: Prentice Hall, 1995.

TODA, H. Y. Statistical inference in vector autoregressions with possibly integrated processes. Journal of Econometrics, p. 225-250, 1995. DOI: https://doi.org/10.1016/03044076(94)01616-8

TSAY, R. S. Analysis of financial time series. Third edition. John Wiley e Sons, 2012.

TSE, Y. K.; Tsui, A. K. A multivariate garch model with timevarying correlations. Available at SSRN 250228, 2000.

VAN, Dijk, D., D. R.; Osborn M. Sensier. Testing for causality in variance in the presence of breaks. Economics Letters 89(2), p. 193-199, 2005. DOI: https://doi.org/10.1016/j.econlet.2005.05.029

$\mathrm{VO}, \mathrm{X}$. International financial integration in asian bond markets. Research in International Business and Finance 23(1), p. 90-106, 2009. DOI: https://doi.org/10.1016/j.ribaf.2008.07.001 


\section{Anexos}

Figura 1: Evolução dos retornos dos ativos analisados
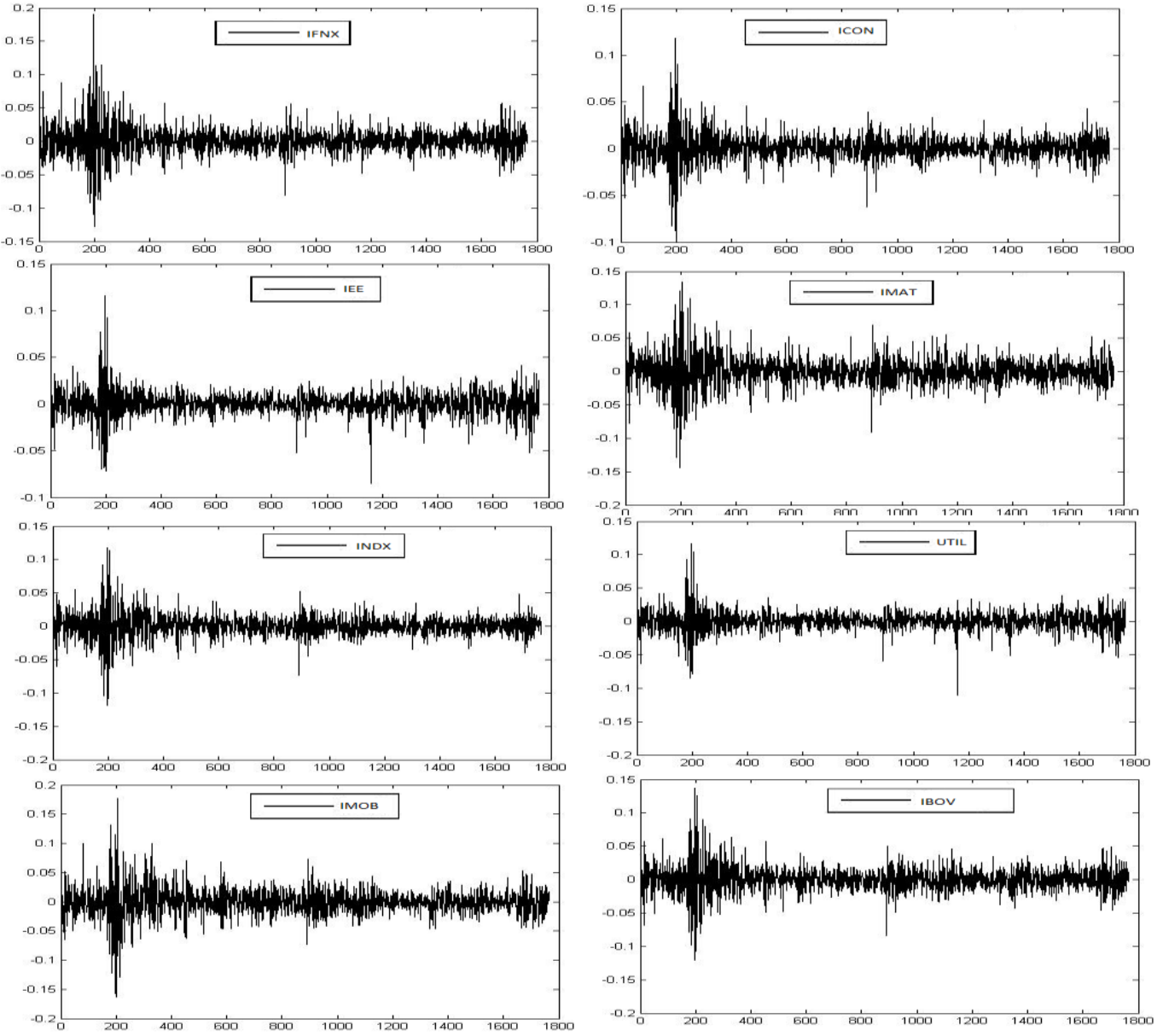

Fonte: Elaboração própria 
Figura 2: Evolução do ajuste nas expectativas de câmbio e Juros

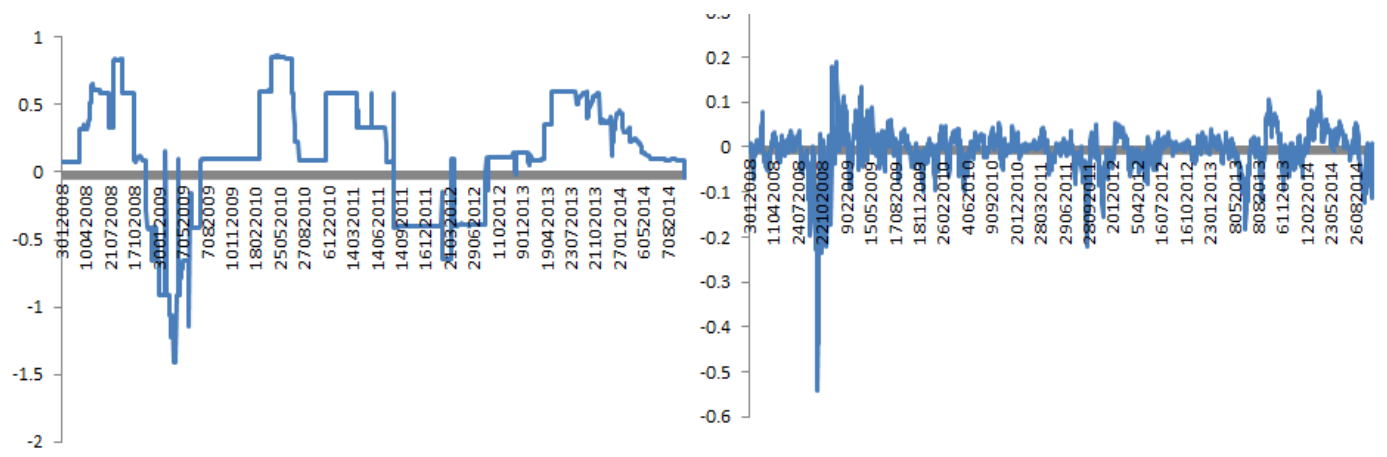

Fonte: Elaboração própria 